(c) the strong triangle inequality holds:

$$
\|x+y\| \leq \max \{\|x\|,\|y\|\}
$$

for all $x, y \in X$.

Then $(X,\|\cdot\|)$ is called a non-Archimedean normed space.

Definition 1.2 Let $\left\{x_{n}\right\}$ be a sequence in a non-Archimedean normed space $X$.

(a) A sequence $\left\{x_{n}\right\}_{n=1}^{\infty}$ in a non-Archimedean space is a Cauchy sequence iff the sequence $\left\{x_{n+1}-x_{n}\right\}_{n=1}^{\infty}$ converges to zero;

(b) The sequence $\left\{x_{n}\right\}$ is said to be convergent if, for any $\varepsilon>0$, there is a positive integer $N$ and $x \in X$ such that $\left\|x_{n}-x\right\| \leq \varepsilon$, for all $n \geq N$. Then the point $x \in X$ is called the limit of the sequence $\left\{x_{n}\right\}$, which is denoted by $\lim _{n \rightarrow \infty} x_{n}=x$;

(c) If every Cauchy sequence in $X$ converges, then the non-Archimedean normed space $X$ is called a non-Archimedean Banach space.

Example 1.1 Fix a prime number $p$. For any nonzero rational number $x$, there exists a unique integer $n_{x} \in \mathbb{Z}$ such that $x=\frac{a}{b} p^{n_{x}}$, where $a$ and $b$ are integers not divisible by $p$. Then $|x|_{p}:=p^{-n_{x}}$ defines a non-Archimedean norm on $\mathbb{Q}$. The completion of $\mathbb{Q}$ with respect to the metric $d(x, y)=|x-y|_{p}$ is denoted by $\mathbb{Q}_{p}$ which is called the $p$-adic number field. In fact, $\mathbb{Q}_{p}$ is the set of all formal series $x=\sum_{k \geq n_{x}}^{\infty} a_{k} p^{k}$ where $\left|a_{k}\right| \leq p-1$ are integers. The addition and multiplication between any two elements of $\mathbb{Q}_{p}$ are defined naturally. The norm $\left|\sum_{k \geq n_{x}}^{\infty} a_{k} p^{k}\right|_{p}=p^{-n_{x}}$ is a non-Archimedean norm on $\mathbb{Q}_{p}$ and it makes $\mathbb{Q}_{p}$ a locally compact field.

Theorem 1.1 Let $(X, d)$ be a complete generalized metric space and $J: X \rightarrow X$ be a strictly contractive mapping with Lipschitz constant $L<1$. Then, for all $x \in X$, either $d\left(J^{n} x, J^{n+1} x\right)=$ $\infty$ for all nonnegative integers $n$ or there exists a positive integer $n_{0}$ such that:

(a) $d\left(J^{n} x, J^{n+1} x\right)<\infty$ for all $n_{0} \geq n_{0}$;

(b) the sequence $\left\{J^{n} x\right\}$ converges to a fixed point $y^{*}$ of $J$;

(c) $y^{\prime \prime}$ is the unique fixed point of $J$ in the set $Y=\left\{y \in X: d\left(J^{n_{0}} x, y\right)<\infty\right\}$;

(d) $d\left(y, y^{*}\right) \leq \frac{1}{1-L} d(y, J y)$ for all $y \in Y$.

In this paper, we prove the generalized Hyers-Ulam stability of the following functional equation:

$$
\sum_{j=1}^{m} f\left(-r_{j} x_{j}+\sum_{1 \leq i \leq m, i \neq j} r_{i} x_{i}\right)+2 \sum_{i=1}^{m} r_{i} f\left(x_{i}\right)=m f\left(\sum_{i=1}^{m} r_{i} x_{i}\right),
$$

where $r_{1}, \ldots, r_{m} \in \mathbb{R}, \sum_{k=1}^{m} r_{k} \neq 0$, and $r_{i}, r_{j} \neq 0$ for some $1 \leq i<j \leq m$, in non-Archimedean Banach spaces. A classical question in the theory of functional equations is the following: 'When is it true that a function which approximately satisfies a functional equation $D$ must be close to an exact solution of $D$ ?'.

If the problem accepts a solution, we say that the equation $D$ is stable. The first stability problem concerning group homomorphisms was raised by Ulam [34] in 1940. 
In the next year D. H. Hyres [17], gave a positive answer to the above question for additive groups under the assumption that the groups are Banach spaces. In 1978, Th. M. Rassias [24] proved a generalization of Hyres' theorem for additive mappings.

The result of Th. M. Rassias has influenced the development of what is now called the Hyers-Ulam-Rassias stability theory for functional equations. In 1994, a generalization of Rassias' theorem was obtained by Gávruta [15] by replacing the bound $\epsilon\left(\|x\|^{p}+\|y\|^{p}\right)$ by a general control function $\varphi(x, y)$.

The stability problems of several functional equations have been extensively investigated by a number of authors and there are many interesting results concerning this problem (see [1-33]).

\section{Non-Archimedean stability of the functional equation (1.1): a fixed point approach}

In this section, using a fixed point alternative approach, we prove the generalized HyersUlam stability of the functional equation (1.1) in non-Archimedean normed spaces. Throughout this section, let $X$ be a non-Archimedean normed space and $Y$ be a nonArchimedean Banach space. Also $|2| \neq 1$.

Lemma 2.1 Let $\mathcal{X}$ and $\mathcal{Y}$ be linear spaces and let $r_{1}, \ldots, r_{n}$ be real numbers with $\sum_{k=1}^{n} r_{k} \neq 0$ and $r_{i}, r_{j} \neq 0$ for some $1 \leq i<j \leq n$. Assume that a mapping $f: \mathcal{X} \rightarrow \mathcal{Y}$ satisfies the functional equation (1.1) for all $x_{1}, \ldots, x_{n} \in \mathcal{X}$. Then the mapping $f$ is Cauchy additive. Moreover, $f\left(r_{k} x\right)=r_{k} f(x)$ for all $x \in \mathcal{X}$ and all $1 \leq k \leq n$.

Proof Since $\sum_{k=1}^{n} r_{k} \neq 0$, putting $x_{1}=\cdots=x_{n}=0$ in (1.1), we get $f(0)=0$. Without loss of generality, we may assume that $r_{1}, r_{2} \neq 0$. Letting $x_{3}=\cdots=x_{n}=0$ in (1.1), we get

$$
f\left(-r_{1} x_{1}+r_{2} x_{2}\right)+f\left(r_{1} x_{1}-r_{2} x_{2}\right)+2 r_{1} f\left(x_{1}\right)+2 r_{2} f\left(x_{2}\right)=2 f\left(r_{1} x_{1}+r_{2} x_{2}\right)
$$

for all $x_{1}, x_{2} \in \mathcal{X}$. Letting $x_{2}=0$ in (2.1), we get

$$
2 r_{1} f\left(x_{1}\right)=f\left(r_{1} x_{1}\right)-f\left(-r_{1} x_{1}\right)
$$

for all $x_{1} \in \mathcal{X}$. Similarly, by putting $x_{1}=0$ in (2.1), we get

$$
2 r_{2} f\left(x_{2}\right)=f\left(r_{2} x_{2}\right)-f\left(-r_{2} x_{2}\right)
$$

for all $x_{1} \in \mathcal{X}$. It follows from (2.1), (2.2) and (2.3) that

$$
\begin{gathered}
f\left(-r_{1} x_{1}+r_{2} x_{2}\right)+f\left(r_{1} x_{1}-r_{2} x_{2}\right)+f\left(r_{1} x_{1}\right)+f\left(r_{2} x_{2}\right) \\
-f\left(-r_{1} x_{1}\right)-f\left(-r_{2} x_{2}\right)=2 f\left(r_{1} x_{1}+r_{2} x_{2}\right)
\end{gathered}
$$

for all $x_{1}, x_{2} \in \mathcal{X}$. Replacing $x_{1}$ and $x_{2}$ by $\frac{x}{r_{1}}$ and $\frac{y}{r_{2}}$ in (2.4), we get

$$
f(-x+y)+f(x-y)+f(x)+f(y)-f(-x)-f(-y)=2 f(x+y)
$$

for all $x, y \in \mathcal{X}$. Letting $y=-x$ in (2.5), we get that $f(-2 x)+f(2 x)=0$ for all $x \in \mathcal{X}$. So the mapping $L$ is odd. Therefore, it follows from (2.5) that the mapping $f$ is additive. Moreover, 
let $x \in \mathcal{X}$ and $1 \leq k \leq n$. Setting $x_{k}=x$ and $x_{l}=0$ for all $1 \leq l \leq n, l \neq k$, in (1.1) and using the oddness of $f$, we get that $f\left(r_{k} x\right)=r_{k} f(x)$.

Using the same method as in the proof of Lemma 2.1, we have an alternative result of Lemma 2.1 when $\sum_{k=1}^{n} r_{k}=0$.

Lemma 2.2 Let $\mathcal{X}$ and $\mathcal{Y}$ be linear spaces and let $r_{1}, \ldots, r_{n}$ be real numbers with $r_{i}, r_{j} \neq$ 0 for some $1 \leq i<j \leq n$. Assume that a mapping $f: \mathcal{X} \rightarrow \mathcal{Y}$ with $f(0)=0$ satisfying the functional equation (1.1) for all $x_{1}, \ldots, x_{n} \in \mathcal{X}$. Then the mapping $f$ is Cauchy additive. Moreover, $f\left(r_{k} x\right)=r_{k} f(x)$ for all $x \in \mathcal{X}$ and all $1 \leq k \leq n$.

Remark 2.1 Throughout this paper, $r_{1}, \ldots, r_{m}$ will be real numbers such that $r_{i}, r_{j} \neq 0$ for fixed $1 \leq i<j \leq m$ and $\varphi_{i, j}(x, y):=\varphi(0, \ldots, 0, \underbrace{x}_{i \text { th }}, 0, \ldots, 0, \underbrace{y}_{j \text { th }}, 0, \ldots, 0)$ for all $x, y \in X$ and all $1 \leq i<j \leq m$.

Theorem 2.1 Let $\varphi: X^{m} \rightarrow[0, \infty)$ be a function such that there exists an $L<1$ with

$$
\varphi\left(\frac{x_{1}}{2}, \ldots, \frac{x_{m}}{2}\right) \leq \frac{L \varphi\left(x_{1}, \ldots, x_{m}\right)}{|2|}
$$

for all $x_{1}, \ldots, x_{m} \in X$. Let $f: X \rightarrow Y$ be a mapping with $f(0)=0$ satisfying the following inequality:

$$
\begin{aligned}
& \left\|\sum_{j=1}^{m} f\left(-r_{j} x_{j}+\sum_{1 \leq i \leq m, i \neq j} r_{i} x_{i}\right)+2 \sum_{i=1}^{m} r_{i} f\left(x_{i}\right)-m f\left(\sum_{i=1}^{m} r_{i} x_{i}\right)\right\| \\
& \quad \leq \varphi\left(x_{1}, \ldots, x_{m}\right)
\end{aligned}
$$

for all $x_{1}, \ldots, x_{m} \in X$. Then there is a unique Euler-Lagrange type additive mapping EL : $X \rightarrow Y$ such that

$$
\begin{aligned}
\|f(x)-E L(x)\| \leq & \frac{L}{|2|-|2| L} \max \left\{\max \left\{\varphi_{i, j}\left(\frac{x}{2 r_{i}},-\frac{x}{2 r_{j}}\right), \varphi_{i, j}\left(\frac{x}{2 r_{i}}, 0\right), \varphi_{i, j}\left(0,-\frac{x}{2 r_{j}}\right)\right\},\right. \\
& \left.\frac{1}{|2|} \max \left\{\varphi_{i, j}\left(\frac{x}{r_{i}}, \frac{x}{r_{j}}\right), \varphi_{i, j}\left(\frac{x}{r_{i}}, 0\right), \varphi_{i, j}\left(0, \frac{x}{r_{j}}\right)\right\}\right\}
\end{aligned}
$$

for all $x \in X$.

Proof For each $1 \leq k \leq m$ with $k \neq i, j$, let $x_{k}=0$ in (2.7). Then we get the following inequality:

$$
\begin{aligned}
& \left\|f\left(-r_{i} x_{i}+r_{j} x_{j}\right)+f\left(r_{i} x_{i}-r_{j} x_{j}\right)-2 f\left(r_{i} x_{i}+r_{j} x_{j}\right)+2 r_{i} f\left(x_{i}\right)+2 r_{j} f\left(x_{j}\right)\right\| \\
& \quad \leq \varphi_{i, j}\left(x_{i}, x_{j}\right)
\end{aligned}
$$

for all $x_{i}, x_{j} \in X$. Letting $x_{i}=0$ in (2.9), we get

$$
\left\|f\left(-r_{j} x_{j}\right)-f\left(r_{j} x_{j}\right)+2 r_{j} f\left(x_{j}\right)\right\| \leq \varphi_{i, j}\left(0, x_{j}\right)
$$


for all $x_{j} \in X$. Similarly, letting $x_{j}=0$ in (2.9), we get

$$
\left\|f\left(-r_{i} x_{i}\right)-f\left(r_{i} x_{i}\right)+2 r_{i} f\left(x_{i}\right)\right\| \leq \varphi_{i, j}\left(x_{i}, 0\right)
$$

for all $x_{i} \in X$. It follows from (2.9), (2.10) and (2.11) that for all $x_{i}, x_{j} \in X$

$$
\begin{aligned}
& \| f\left(-r_{i} x_{i}+r_{j} x_{j}\right)+f\left(r_{i} x_{i}-r_{j} x_{j}\right)-2 f\left(r_{i} x_{i}+r_{j} x_{j}\right)+2 r_{i} f\left(x_{i}\right)+2 r_{j} f\left(x_{j}\right) \\
& \quad-\left(f\left(-r_{i} x_{i}\right)-f\left(r_{i} x_{i}\right)+2 r_{i} f\left(x_{i}\right)\right)-\left(f\left(-r_{j} x_{j}\right)-f\left(r_{j} x_{j}\right)+2 r_{j} f\left(x_{j}\right)\right) \| \\
& \leq \max \left\{\varphi_{i, j}\left(x_{i}, x_{j}\right), \varphi_{i, j}\left(0, x_{j}\right), \varphi_{i, j}\left(x_{i}, 0\right)\right\} .
\end{aligned}
$$

Replacing $x_{i}$ and $x_{j}$ by $\frac{x}{r_{i}}$ and $\frac{y}{r_{j}}$ in (2.12), we get that

$$
\begin{aligned}
& \|f(-x+y)+f(x-y)-2 f(x+y)+f(x)+f(y)-f(-x)-f(-y)\| \\
& \quad \leq \max \left\{\varphi_{i, j}\left(\frac{x}{r_{i}}, \frac{y}{r_{j}}\right), \varphi_{i, j}\left(\frac{x}{r_{i}}, 0\right), \varphi_{i, j}\left(0, \frac{y}{r_{j}}\right)\right\}
\end{aligned}
$$

for all $x, y \in X$. Putting $y=x$ in (2.13), we get

$$
\|f(x)-f(-x)-f(2 x)\| \leq \frac{1}{|2|} \max \left\{\varphi_{i, j}\left(\frac{x}{r_{i}}, \frac{x}{r_{j}}\right), \varphi_{i, j}\left(\frac{x}{r_{i}}, 0\right), \varphi_{i, j}\left(0, \frac{x}{r_{j}}\right)\right\}
$$

for all $x \in X$. Replacing $x$ and $y$ by $\frac{x}{2}$ and $-\frac{x}{2}$ in (2.13) respectively, we get

$$
\|f(x)+f(-x)\| \leq \max \left\{\varphi_{i, j}\left(\frac{x}{2 r_{i}},-\frac{x}{2 r_{j}}\right), \varphi_{i, j}\left(\frac{x}{2 r_{i}}, 0\right), \varphi_{i, j}\left(0,-\frac{x}{2 r_{j}}\right)\right\}
$$

for all $x \in X$. It follows from (2.14) and (2.15) that

$$
\begin{aligned}
\|f(2 x)-2 f(x)\| \\
\quad=\|f(x)+f(-x)+f(x)-f(-x)-f(2 x)\| \\
\quad \leq \max \left\{\max \left\{\varphi_{i, j}\left(\frac{x}{2 r_{i}},-\frac{x}{2 r_{j}}\right), \varphi_{i, j}\left(\frac{x}{2 r_{i}}, 0\right), \varphi_{i, j}\left(0,-\frac{x}{2 r_{j}}\right)\right\},\right. \\
\left.\quad \frac{1}{|2|} \max \left\{\varphi_{i, j}\left(\frac{x}{r_{i}}, \frac{x}{r_{j}}\right), \varphi_{i, j}\left(\frac{x}{r_{i}}, 0\right), \varphi_{i, j}\left(0, \frac{x}{r_{j}}\right)\right\}\right\}
\end{aligned}
$$

for all $x \in X$. Replacing $x$ by $\frac{x}{2}$ in (2.16), we obtain

$$
\begin{aligned}
& \left\|f(x)-2 f\left(\frac{x}{2}\right)\right\| \\
& \leq \max \left\{\max \left\{\varphi_{i, j}\left(\frac{x}{4 r_{i}},-\frac{x}{4 r_{j}}\right), \varphi_{i, j}\left(\frac{x}{4 r_{i}}, 0\right), \varphi_{i, j}\left(0,-\frac{x}{4 r_{j}}\right)\right\},\right. \\
& \left.\quad \frac{1}{|2|} \max \left\{\varphi_{i, j}\left(\frac{x}{2 r_{i}}, \frac{x}{2 r_{j}}\right), \varphi_{i, j}\left(\frac{x}{2 r_{i}}, 0\right), \varphi_{i, j}\left(0, \frac{x}{2 r_{j}}\right)\right\}\right\} .
\end{aligned}
$$


Consider the set $S:=\{g: X \rightarrow Y ; g(0)=0\}$ and the generalized metric $d$ in $S$ defined by

$$
\begin{aligned}
d(f, g)= & \inf _{\mu \in \mathbb{R}^{+}}\left\{\|g(x)-h(x)\| \leq \mu \max \left\{\operatorname { m a x } \left\{\varphi_{i, j}\left(\frac{x}{2 r_{i}},-\frac{x}{2 r_{j}}\right),\right.\right.\right. \\
& \left.\varphi_{i, j}\left(\frac{x}{2 r_{i}}, 0\right), \varphi_{i, j}\left(0,-\frac{x}{2 r_{j}}\right)\right\}, \\
& \left.\left.\frac{1}{|2|} \max \left\{\varphi_{i, j}\left(\frac{x}{r_{i}}, \frac{x}{r_{j}}\right), \varphi_{i, j}\left(\frac{x}{r_{i}}, 0\right), \varphi_{i, j}\left(0, \frac{x}{r_{j}}\right)\right\}\right\}, \forall x \in X\right\},
\end{aligned}
$$

where $\inf \emptyset=+\infty$. It is easy to show that $(S, d)$ is complete (see [18], Lemma 2.1). Now, we consider a linear mapping $J: S \rightarrow S$ such that

$$
J h(x):=2 h\left(\frac{x}{2}\right)
$$

for all $x \in X$. Let $g, h \in S$ be such that $d(g, h)=\epsilon$. Then

$$
\begin{aligned}
\|g(x)-h(x)\| \leq & \epsilon \max \left\{\max \left\{\varphi_{i, j}\left(\frac{x}{2 r_{i}},-\frac{x}{2 r_{j}}\right), \varphi_{i, j}\left(\frac{x}{2 r_{i}}, 0\right), \varphi_{i, j}\left(0,-\frac{x}{2 r_{j}}\right)\right\},\right. \\
& \left.\frac{1}{|2|} \max \left\{\varphi_{i, j}\left(\frac{x}{r_{i}}, \frac{x}{r_{j}}\right), \varphi_{i, j}\left(\frac{x}{r_{i}}, 0\right), \varphi_{i, j}\left(0, \frac{x}{r_{j}}\right)\right\}\right\}
\end{aligned}
$$

for all $x \in X$, and so

$$
\begin{aligned}
\|J g(x)-J h(x)\|= & \left\|2 g\left(\frac{x}{2}\right)-2 h\left(\frac{x}{2}\right)\right\| \\
\leq & |2| \epsilon \max \left\{\max \left\{\varphi_{i, j}\left(\frac{x}{4 r_{i}},-\frac{x}{4 r_{j}}\right), \varphi_{i, j}\left(\frac{x}{4 r_{i}}, 0\right), \varphi_{i, j}\left(0,-\frac{x}{4 r_{j}}\right)\right\},\right. \\
& \left.\frac{1}{|2|} \max \left\{\varphi_{i, j}\left(\frac{x}{2 r_{i}}, \frac{x}{2 r_{j}}\right), \varphi_{i, j}\left(\frac{x}{2 r_{i}}, 0\right), \varphi_{i, j}\left(0, \frac{x}{2 r_{j}}\right)\right\}\right\} \\
\leq & |2| \frac{L \epsilon}{|2|} \max \left\{\max \left\{\varphi_{i, j}\left(\frac{x}{2 r_{i}},-\frac{x}{2 r_{j}}\right), \varphi_{i, j}\left(\frac{x}{2 r_{i}}, 0\right), \varphi_{i, j}\left(0,-\frac{x}{2 r_{j}}\right)\right\},\right. \\
& \left.\frac{1}{|2|} \max \left\{\varphi_{i, j}\left(\frac{x}{r_{i}}, \frac{x}{r_{j}}\right), \varphi_{i, j}\left(\frac{x}{r_{i}}, 0\right), \varphi_{i, j}\left(0, \frac{x}{r_{j}}\right)\right\}\right\}
\end{aligned}
$$

for all $x \in X$. Thus $d(g, h)=\epsilon$ implies that $d(J g, J h) \leq L \epsilon$. This means that

$$
d(J g, J h) \leq L d(g, h)
$$

for all $g, h \in S$. It follows from (2.17) that

$$
d(f, J f) \leq \frac{L}{|2|} .
$$

By Theorem 1.1, there exists a mapping $E L: X \rightarrow Y$ satisfying the following:

(1) $E L$ is a fixed point of $J$, that is,

$$
E L\left(\frac{x}{2}\right)=\frac{1}{2} E L(x)
$$


for all $x \in X$. The mapping $E L$ is a unique fixed point of $J$ in the set

$$
\Omega=\{h \in S: d(g, h)<\infty\} .
$$

This implies that $E L$ is a unique mapping satisfying (2.18) such that there exists $\mu \in(0, \infty)$ satisfying

$$
\begin{aligned}
\|f(x)-E L(x)\| \leq & \mu \max \left\{\max \left\{\varphi_{i, j}\left(\frac{x}{2 r_{i}},-\frac{x}{2 r_{j}}\right), \varphi_{i, j}\left(\frac{x}{2 r_{i}}, 0\right), \varphi_{i, j}\left(0,-\frac{x}{2 r_{j}}\right)\right\},\right. \\
& \left.\frac{1}{|2|} \max \left\{\varphi_{i, j}\left(\frac{x}{r_{i}}, \frac{x}{r_{j}}\right), \varphi_{i, j}\left(\frac{x}{r_{i}}, 0\right), \varphi_{i, j}\left(0, \frac{x}{r_{j}}\right)\right\}\right\}
\end{aligned}
$$

for all $x \in X$.

(2) $d\left(J^{n} f, E L\right) \rightarrow 0$ as $n \rightarrow \infty$. This implies the equality

$$
\lim _{n \rightarrow \infty} 2^{n} f\left(\frac{x}{2^{n}}\right)=E L(x)
$$

for all $x \in X$.

(3) $d(f, E L) \leq \frac{d(f, J f)}{1-L}$ with $f \in \Omega$, which implies the inequality

$$
d(f, E L) \leq \frac{L}{|2|-|2| L}
$$

This implies that the inequality (2.8) holds.

By (2.6) and (2.7), we obtain

$$
\begin{aligned}
& \left\|\sum_{j=1}^{m} E L\left(-r_{j} x_{j}+\sum_{1 \leq i \leq m, i \neq j} r_{i} x_{i}\right)+2 \sum_{i=1}^{m} r_{i} E L\left(x_{i}\right)-m E L\left(\sum_{i=1}^{m} r_{i} x_{i}\right)\right\| \\
& \quad=\lim _{n \rightarrow \infty}|2|^{n}\left\|\sum_{j=1}^{m} f\left(\frac{-r_{j} x_{j}}{2^{n}}+\sum_{1 \leq i \leq m, i \neq j} \frac{r_{i} x_{i}}{2^{n}}\right)+2 \sum_{i=1}^{m} r_{i} f\left(\frac{x_{i}}{2^{n}}\right)-m f\left(\sum_{i=1}^{m} \frac{r_{i} x_{i}}{2^{n}}\right)\right\| \\
& \quad \leq \lim _{n \rightarrow \infty}|2|^{n} \varphi\left(\frac{x_{1}}{2^{n}}, \ldots, \frac{x_{m}}{2^{n}}\right) \\
& \quad \leq \lim _{n \rightarrow \infty}|2|^{n} \cdot \frac{L^{n}}{|2|^{n}} \varphi\left(x_{1}, \ldots, x_{m}\right)
\end{aligned}
$$

for all $x_{1}, \ldots, x_{m} \in X$ and $n \in \mathbb{N}$. So $E L$ satisfies (1.1). Thus, the mapping $E L: X \rightarrow Y$ is Euler-Lagrange type additive, as desired.

Corollary 2.1 Let $\theta \geq 0$ and $r$ be a real number with $0<r<1$. Let $f: X \rightarrow Y$ be a mapping with $f(0)=0$ satisfying the inequality

$$
\begin{aligned}
& \left\|\sum_{j=1}^{m} f\left(-r_{j} x_{j}+\sum_{1 \leq i \leq m, i \neq j} r_{i} x_{i}\right)+2 \sum_{i=1}^{m} r_{i} f\left(x_{i}\right)-m f\left(\sum_{i=1}^{m} r_{i} x_{i}\right)\right\| \\
& \quad \leq \theta\left(\sum_{i=1}^{m}\left\|x_{i}\right\|^{r}\right)
\end{aligned}
$$


for all $x_{1}, \ldots, x \in X$. Then, the limit $E L(x)=\lim _{n \rightarrow \infty} 2^{n} f\left(\frac{x}{2^{n}}\right)$ exists for all $x \in X$ and $E L$ : $X \rightarrow Y$ is a unique Euler-Lagrange additive mapping such that

$$
\begin{aligned}
\|f(x)-E L(x)\| \leq & \frac{|2|}{|2|^{r+1}-|2|^{2}} \max \left\{\max \left\{\frac{|2|^{r} \theta\|x\|^{r}\left(\left|r_{i}\right|^{r}+\left|r_{j}\right|^{r}\right)}{|4|^{r}\left|r_{i} r_{j}\right|^{r}}, \frac{\theta\|x\|^{r}}{|2|^{r}\left|r_{i}\right|^{\mid}}, \frac{\theta\|x\|^{r}}{|2|^{r}\left|r_{j}\right|^{r}}\right\},\right. \\
& \left.\frac{1}{|2|} \max \left\{\frac{\theta\|x\|^{r}\left(\left|r_{i}\right|^{r}+\left|r_{j}\right|^{r}\right)}{\left|r_{i} r_{j}\right|^{r}}, \frac{\theta\|x\|^{r}}{\left|r_{i}\right|^{r}}, \frac{\theta\|x\|^{r}}{\left|r_{j}\right|^{r}}\right\}\right\} \\
\leq & \frac{\theta\|x\|^{r}\left(\left|r_{i}\right|^{r}+\left|r_{j}\right|^{r}\right)}{\left|r_{i} r_{j}\right|^{r}\left(|2|^{r+1}-|2|^{2}\right)}
\end{aligned}
$$

for all $x \in X$.

Proof The proof follows from Theorem 2.1 by taking $\varphi\left(x_{1}, \ldots, x_{m}\right)=\theta\left(\sum_{i=1}^{m}\left\|x_{i}\right\|^{r}\right)$ for all $x_{1}, \ldots, x_{m} \in X$. In fact, if we choose $L=|2|^{1-r}$, then we get the desired result.

Theorem 2.2 Let $\varphi: X^{m} \rightarrow[0, \infty)$ be a function such that there exists an $L<1$ with

$$
\varphi\left(x_{1}, \ldots, x_{m}\right) \leq|2| L \varphi\left(\frac{x_{1}}{2}, \ldots, \frac{x_{m}}{2}\right)
$$

for all $x_{1}, \ldots, x_{m} \in X$. Let $f: X \rightarrow Y$ be a mapping with $f(0)=0$ satisfying the inequality (2.7). Then, there is a unique Euler-Lagrange additive mapping $E L: X \rightarrow Y$ such that

$$
\begin{aligned}
\|f(x)-E L(x)\| \leq & \frac{1}{|2|-|2| L} \max \left\{\max \left\{\varphi_{i, j}\left(\frac{x}{2 r_{i}},-\frac{x}{2 r_{j}}\right), \varphi_{i, j}\left(\frac{x}{2 r_{i}}, 0\right), \varphi_{i, j}\left(0,-\frac{x}{2 r_{j}}\right)\right\}\right. \\
& \left.\frac{1}{|2|} \max \left\{\varphi_{i, j}\left(\frac{x}{r_{i}}, \frac{x}{r_{j}}\right), \varphi_{i, j}\left(\frac{x}{r_{i}}, 0\right), \varphi_{i, j}\left(0, \frac{x}{r_{j}}\right)\right\}\right\}
\end{aligned}
$$

Proof By (2.16), we have

$$
\begin{aligned}
\left\|\frac{f(2 x)}{2}-f(x)\right\| \leq & \frac{1}{|2|} \max \left\{\max \left\{\varphi_{i, j}\left(\frac{x}{2 r_{i}},-\frac{x}{2 r_{j}}\right), \varphi_{i, j}\left(\frac{x}{2 r_{i}}, 0\right), \varphi_{i, j}\left(0,-\frac{x}{2 r_{j}}\right)\right\},\right. \\
& \left.\frac{1}{|2|} \max \left\{\varphi_{i, j}\left(\frac{x}{r_{i}}, \frac{x}{r_{j}}\right), \varphi_{i, j}\left(\frac{x}{r_{i}}, 0\right), \varphi_{i, j}\left(0, \frac{x}{r_{j}}\right)\right\}\right\}
\end{aligned}
$$

for all $x \in X$. Let $(S, d)$ be the generalized metric space defined in the proof of Theorem 2.1. Now, we consider a linear mapping $J: S \rightarrow S$ such that

$$
J h(x):=\frac{1}{2} h(2 x)
$$

for all $x \in X$. Let $g, h \in S$ be such that $d(g, h)=\epsilon$. Then

$$
\begin{aligned}
\|g(x)-h(x)\| \leq & \epsilon \max \left\{\max \left\{\varphi_{i, j}\left(\frac{x}{2 r_{i}},-\frac{x}{2 r_{j}}\right), \varphi_{i, j}\left(\frac{x}{2 r_{i}}, 0\right), \varphi_{i, j}\left(0,-\frac{x}{2 r_{j}}\right)\right\},\right. \\
& \left.\frac{1}{|2|} \max \left\{\varphi_{i, j}\left(\frac{x}{r_{i}}, \frac{x}{r_{j}}\right), \varphi_{i, j}\left(\frac{x}{r_{i}}, 0\right), \varphi_{i, j}\left(0, \frac{x}{r_{j}}\right)\right\}\right\}
\end{aligned}
$$


for all $x \in X$, and so

$$
\begin{aligned}
\|g(x)-J h(x)\|= & \left\|\frac{g(2 x)}{2}-\frac{h(2 x)}{2}\right\| \\
\leq & \frac{1}{|2|} \epsilon \max \left\{\max \left\{\varphi_{i, j}\left(\frac{x}{r_{i}},-\frac{x}{r_{j}}\right), \varphi_{i, j}\left(\frac{x}{r_{i}}, 0\right), \varphi_{i, j}\left(0,-\frac{x}{r_{j}}\right)\right\},\right. \\
& \left.\frac{1}{|2|} \max \left\{\varphi_{i, j}\left(\frac{2 x}{r_{i}}, \frac{2 x}{r_{j}}\right), \varphi_{i, j}\left(\frac{2 x}{r_{i}}, 0\right), \varphi_{i, j}\left(0, \frac{2 x}{r_{j}}\right)\right\}\right\} \\
\leq & |2| L \frac{\epsilon}{|2|} \max \left\{\max \left\{\varphi_{i, j}\left(\frac{x}{2 r_{i}},-\frac{x}{2 r_{j}}\right), \varphi_{i, j}\left(\frac{x}{2 r_{i}}, 0\right), \varphi_{i, j}\left(0,-\frac{x}{2 r_{j}}\right)\right\},\right. \\
& \left.\frac{1}{|2|} \max \left\{\varphi_{i, j}\left(\frac{x}{r_{i}}, \frac{x}{r_{j}}\right), \varphi_{i, j}\left(\frac{x}{r_{i}}, 0\right), \varphi_{i, j}\left(0, \frac{x}{r_{j}}\right)\right\}\right\}
\end{aligned}
$$

for all $x \in X$. Thus $d(g, h)=\epsilon$ implies that $d(J g, J h) \leq L \epsilon$. This means that

$$
d(J g, J h) \leq L d(g, h)
$$

for all $g, h \in S$. It follows from (2.23) that

$$
d(f, J f) \leq \frac{1}{|2|}
$$

By Theorem 1.1, there exists a mapping $E L: X \rightarrow Y$ satisfying the following:

(1) $E L$ is a fixed point of $J$, that is,

$$
E L(2 x)=2 E L(x)
$$

for all $x \in X$. The mapping $E L$ is a unique fixed point of $J$ in the set

$$
\Omega=\{h \in S: d(g, h)<\infty\}
$$

This implies that $E L$ is a unique mapping satisfying (2.24) such that there exists $\mu \in(0, \infty)$ satisfying

$$
\begin{aligned}
\|g(x)-h(x)\| \leq & \mu \max \left\{\max \left\{\varphi_{i, j}\left(\frac{x}{2 r_{i}},-\frac{x}{2 r_{j}}\right), \varphi_{i, j}\left(\frac{x}{2 r_{i}}, 0\right), \varphi_{i, j}\left(0,-\frac{x}{2 r_{j}}\right)\right\},\right. \\
& \left.\frac{1}{|2|} \max \left\{\varphi_{i, j}\left(\frac{x}{r_{i}}, \frac{x}{r_{j}}\right), \varphi_{i, j}\left(\frac{x}{r_{i}}, 0\right), \varphi_{i, j}\left(0, \frac{x}{r_{j}}\right)\right\}\right\}
\end{aligned}
$$

for all $x \in X$.

(2) $d\left(J^{n} f, E L\right) \rightarrow 0$ as $n \rightarrow \infty$. This implies the equality

$$
\lim _{n \rightarrow \infty} \frac{f\left(2^{n} x\right)}{2^{n}}=E L(x)
$$

for all $x \in X$. 
(3) $d(f, E L) \leq \frac{d(f, J f)}{1-L}$ with $f \in \Omega$, which implies the inequality

$$
d(f, E L) \leq \frac{1}{|2|-|2| L}
$$

This implies that the inequality (2.22) holds. The rest of the proof is similar to the proof of Theorem 2.1 .

Corollary 2.2 Let $\theta \geq 0$ and $r$ be a real number with $r>1$. Let $f: X \rightarrow Y$ be a mapping with $f(0)=0$ satisfying (2.20). Then, the limit $E L(x)=\lim _{n \rightarrow \infty} \frac{f\left(2^{n} x\right)}{2^{n}}$ exists for all $x \in X$ and $E L: X \rightarrow Y$ is a unique cubic mapping such that

$$
\begin{aligned}
\|f(x)-E L(x)\| \leq & \frac{1}{|2|-|2|^{r}} \max \left\{\max \left\{\frac{|2|^{r} \theta\|x\|^{r}\left(\left|r_{i}\right|^{r}+\left|r_{j}\right|^{r}\right)}{|4|^{r}\left|r_{i} r_{j}\right|^{r}}, \frac{\theta\|x\|^{r}}{|2|^{r}\left|r_{i}\right|^{r}}, \frac{\theta\|x\|^{r}}{|2|^{r}\left|r_{j}\right|^{r}}\right\},\right. \\
& \left.\frac{1}{|2|} \max \left\{\frac{\theta\|x\|^{r}\left(\left|r_{i}\right|^{r}+\left|r_{j}\right|^{r}\right)}{\left|r_{i} r_{j}\right|^{r}}, \frac{\theta\|x\|^{r}}{\left|r_{i}\right|^{r}}, \frac{\theta\|x\|^{r}}{\left|r_{j}\right|^{r}}\right\}\right\} \leq \frac{\theta\|x\|^{r}\left(\left|r_{i}\right|^{r}+\left|r_{j}\right|^{r}\right)}{\left|r_{i} r_{j}\right|^{r}\left(|2|^{r+1}-|2|^{r+2}\right)}
\end{aligned}
$$

for all $x \in X$.

Proof The proof follows from Theorem 2.2 by taking $\varphi\left(x_{1}, \ldots, x_{m}\right)=\theta\left(\sum_{i=1}^{m}\left\|x_{i}\right\|^{r}\right)$ for all $x_{1}, \ldots, x_{m} \in X$. In fact, if we choose $L=|2|^{r-1}$, then we get the desired result.

\section{Non-Archimedean stability of the functional equation (1.1): a direct method}

In this section, using a direct method, we prove the generalized Hyers-Ulam stability of the cubic functional equation (1.1) in non-Archimedean normed spaces. Throughout this section, we assume that $G$ is an additive semigroup and $X$ is a non-Archimedean Banach space.

Theorem 3.1 Let $\varphi: G^{m} \rightarrow[0,+\infty)$ be a function such that

$$
\lim _{n \rightarrow \infty}|2|^{n} \varphi\left(\frac{x_{1}}{2^{n}}, \ldots, \frac{x_{m}}{2^{n}}\right)=0
$$

for all $x_{1}, \ldots, x_{m} \in G$ and let for each $x \in G$ the limit

$$
\begin{aligned}
\Theta(x)= & \lim _{n \rightarrow \infty} \max \left\{| 2 | ^ { k } \operatorname { m a x } \left\{\operatorname { m a x } \left\{\varphi_{i, j}\left(\frac{x}{2^{k+2} r_{i}},-\frac{x}{2^{k+2} r_{j}}\right), \varphi_{i, j}\left(\frac{x}{2^{k+2} r_{i}}, 0\right),\right.\right.\right. \\
& \left.\varphi_{i, j}\left(0,-\frac{x}{2^{k+2} r_{j}}\right)\right\}, \frac{1}{|2|} \max \left\{\varphi_{i, j}\left(\frac{x}{2^{k+1} r_{i}}, \frac{x}{2^{k+1} r_{j}}\right), \varphi_{i, j}\left(\frac{x}{2^{k+1} r_{i}}, 0\right),\right. \\
& \left.\left.\left.\varphi_{i, j}\left(0, \frac{x}{2^{k+1} r_{j}}\right)\right\}\right\} \mid 0 \leq k<n\right\}
\end{aligned}
$$

exist. Suppose that $f: G \rightarrow X$ is a mapping with $f(0)=0$ satisfying the following inequality:

$$
\begin{aligned}
& \left\|\sum_{j=1}^{m} f\left(-r_{j} x_{j}+\sum_{1 \leq i \leq m, i \neq j} r_{i} x_{i}\right)+2 \sum_{i=1}^{m} r_{i} f\left(x_{i}\right)-m f\left(\sum_{i=1}^{m} r_{i} x_{i}\right)\right\| \\
& \quad \leq \varphi\left(x_{1}, \ldots, x_{m}\right)
\end{aligned}
$$


for all $x_{1}, \ldots, x_{m} \in X$. Then, the limit $E L(x):=\lim _{n \rightarrow \infty} 2^{n} f\left(\frac{x}{2^{n}}\right)$ existsfor all $x \in G$ and defines an Euler-Lagrange type additive mapping $E L: G \rightarrow X$ such that

$$
\|f(x)-E L(x)\| \leq \Theta(x)
$$

Moreover, if

$$
\begin{aligned}
& \lim _{p \rightarrow \infty} \lim _{n \rightarrow \infty} \max \left\{| 2 | ^ { k } \operatorname { m a x } \left\{\operatorname { m a x } \left\{\varphi_{i, j}\left(\frac{x}{2^{k+2} r_{i}},-\frac{x}{2^{k+2} r_{j}}\right), \varphi_{i, j}\left(\frac{x}{2^{k+2} r_{i}}, 0\right),\right.\right.\right. \\
& \left.\varphi_{i, j}\left(0,-\frac{x}{2^{k+2} r_{j}}\right)\right\}, \frac{1}{|2|} \max \left\{\varphi_{i, j}\left(\frac{x}{2^{k+1} r_{i}}, \frac{x}{2^{k+1} r_{j}}\right), \varphi_{i, j}\left(\frac{x}{2^{k+1} r_{i}}, 0\right),\right. \\
& \left.\left.\left.\varphi_{i, j}\left(0, \frac{x}{2^{k+1} r_{j}}\right)\right\}\right\} \mid p \leq k<n+p\right\}
\end{aligned}
$$

then EL is the unique mapping satisfying (3.4).

Proof By (2.17), we know

$$
\begin{aligned}
\left\|f(x)-2 f\left(\frac{x}{2}\right)\right\| \leq & \max \left\{\max \left\{\varphi_{i, j}\left(\frac{x}{4 r_{i}},-\frac{x}{4 r_{j}}\right), \varphi_{i, j}\left(\frac{x}{4 r_{i}}, 0\right), \varphi_{i, j}\left(0,-\frac{x}{4 r_{j}}\right)\right\},\right. \\
& \left.\frac{1}{|2|} \max \left\{\varphi_{i, j}\left(\frac{x}{2 r_{i}}, \frac{x}{2 r_{j}}\right), \varphi_{i, j}\left(\frac{x}{2 r_{i}}, 0\right), \varphi_{i, j}\left(0, \frac{x}{2 r_{j}}\right)\right\}\right\}
\end{aligned}
$$

for all $x \in G$. Replacing $x$ by $\frac{x}{2^{n}}$ in (3.5), we obtain

$$
\begin{aligned}
\left\|2^{n} f\left(\frac{x}{2^{n}}\right)-2^{n+1} f\left(\frac{x}{2^{n+1}}\right)\right\| \\
\leq|2|^{n} \max \left\{\max \left\{\varphi_{i, j}\left(\frac{x}{2^{n+2} r_{i}},-\frac{x}{2^{n+2} r_{j}}\right), \varphi_{i, j}\left(\frac{x}{2^{n+2} r_{i}}, 0\right), \varphi_{i, j}\left(0,-\frac{x}{2^{n+2} r_{j}}\right)\right\},\right. \\
\left.\quad \frac{1}{|2|} \max \left\{\varphi_{i, j}\left(\frac{x}{2^{n+1} r_{i}}, \frac{x}{2^{n+1} r_{j}}\right), \varphi_{i, j}\left(\frac{x}{2^{n+1} r_{i}}, 0\right), \varphi_{i, j}\left(0, \frac{x}{2^{n+1} r_{j}}\right)\right\}\right\} .
\end{aligned}
$$

It follows from (3.1) and (3.6) that the sequence $\left\{2^{n} f\left(\frac{x}{2^{n}}\right)\right\}_{n \geq 1}$ is a Cauchy sequence. Since $X$ is complete, so $\left\{2^{n} f\left(\frac{x}{2^{n}}\right)\right\}_{n \geq 1}$ is convergent. Set

$$
E L(x):=\lim _{n \rightarrow \infty} 2^{n} f\left(\frac{x}{2^{n}}\right)
$$

Using induction on $n$, one can show that

$$
\begin{aligned}
\left\|2^{n} f\left(\frac{x}{2^{n}}\right)-f(x)\right\| \\
\leq \max \left\{| 2 | ^ { k } \operatorname { m a x } \left\{\max \left\{\varphi_{i, j}\left(\frac{x}{2^{k+2} r_{i}},-\frac{x}{2^{k+2} r_{j}}\right), \varphi_{i, j}\left(\frac{x}{2^{k+2} r_{i}}, 0\right), \varphi_{i, j}\left(0,-\frac{x}{2^{k+2} r_{j}}\right)\right\},\right.\right. \\
\left.\left.\quad \frac{1}{|2|} \max \left\{\varphi_{i, j}\left(\frac{x}{2^{k+1} r_{i}}, \frac{x}{2^{k+1} r_{j}}\right), \varphi_{i, j}\left(\frac{x}{2^{k+1} r_{i}}, 0\right), \varphi_{i, j}\left(0, \frac{x}{2^{k+1} r_{j}}\right)\right\}\right\} \mid 0 \leq k<n\right\},
\end{aligned}
$$


for all $n \in \mathbb{N}$ and all $x \in G$. By taking $n$ to approach infinity in (3.7) and using (3.2), one obtains (3.4). By (3.1) and (3.3), we get

$$
\begin{aligned}
& \left\|\sum_{j=1}^{m} E L\left(-r_{j} x_{j}+\sum_{1 \leq i \leq m, i \neq j} r_{i} x_{i}\right)+2 \sum_{i=1}^{m} r_{i} E L\left(x_{i}\right)-m E L\left(\sum_{i=1}^{m} r_{i} x_{i}\right)\right\| \\
& \quad=\lim _{n \rightarrow \infty}|2|^{n}\left\|\sum_{j=1}^{m} f\left(\frac{-r_{j} x_{j}}{2^{n}}+\sum_{1 \leq i \leq m, i \neq j} \frac{r_{i} x_{i}}{2^{n}}\right)+2 \sum_{i=1}^{m} r_{i} f\left(\frac{x_{i}}{2^{n}}\right)-m f\left(\sum_{i=1}^{m} \frac{r_{i} x_{i}}{2^{n}}\right)\right\| \\
& \quad \leq \lim _{n \rightarrow \infty}|2|^{n} \varphi\left(\frac{x_{1}}{2^{n}}, \ldots, \frac{x_{m}}{2^{n}}\right) \\
& \quad=0
\end{aligned}
$$

for all $x_{1}, \ldots, x_{m} \in X$. Therefore the function $E L: G \rightarrow X$ satisfies (1.1).

To prove the uniqueness property of $E L$, let $A: G \rightarrow X$ be another function satisfying (3.4). Then

$$
\begin{aligned}
&\|E L(x)-A(x)\| \\
&=\lim _{j \rightarrow \infty}|2|^{j}\left\|E L\left(\frac{x}{2^{j}}\right)-A\left(\frac{x}{2^{j}}\right)\right\| \\
& \leq \lim _{j \rightarrow \infty}|2|^{j} \max \left\{\left\|E L\left(\frac{x}{2^{j}}\right)-f\left(\frac{x}{2^{j}}\right)\right\|,\left\|f\left(\frac{x}{2^{j}}\right)-A\left(\frac{x}{2^{j}}\right)\right\|\right\} \\
& \leq \lim _{j \rightarrow \infty} \lim _{n \rightarrow \infty} \max \left\{| 2 | ^ { k } \operatorname { m a x } \left\{\operatorname { m a x } \left\{\varphi_{i, j}\left(\frac{x}{2^{k+2} r_{i}},-\frac{x}{2^{k+2} r_{j}}\right), \varphi_{i, j}\left(\frac{x}{2^{k+2} r_{i}}, 0\right),\right.\right.\right. \\
&\left.\varphi_{i, j}\left(0,-\frac{x}{2^{k+2} r_{j}}\right)\right\}, \frac{1}{|2|} \max \left\{\varphi_{i, j}\left(\frac{x}{2^{k+1} r_{i}}, \frac{x}{2^{k+1} r_{j}}\right), \varphi_{i, j}\left(\frac{x}{2^{k+1} r_{i}}, 0\right),\right. \\
&\left.\left.\left.\varphi_{i, j}\left(0, \frac{x}{2^{k+1} r_{j}}\right)\right\}\right\} \mid j \leq k<n+j\right\} \\
&= 0
\end{aligned}
$$

for all $x \in G$. Therefore $A=E L$, and the proof is complete.

Corollary 3.1 Let $\xi:[0, \infty) \rightarrow[0, \infty)$ be a function satisfying

$$
\xi\left(\frac{t}{|2|}\right) \leq \xi\left(\frac{1}{|2|}\right) \xi(t) \quad(t \geq 0) \quad \xi\left(\frac{1}{|2|}\right)<|2|^{-1}
$$

Let $\kappa>0$ and $f: G \rightarrow X$ be a mapping with $f(0)=0$ satisfying the following inequality:

$$
\begin{aligned}
& \left\|\sum_{j=1}^{m} f\left(-r_{j} x_{j}+\sum_{1 \leq i \leq m, i \neq j} r_{i} x_{i}\right)+2 \sum_{i=1}^{m} r_{i} f\left(x_{i}\right)-m f\left(\sum_{i=1}^{m} r_{i} x_{i}\right)\right\| \\
& \quad \leq \kappa\left(\sum_{k=1}^{m} \xi\left(\left|x_{k}\right|\right)\right)
\end{aligned}
$$


for all $x_{1}, \ldots, x_{m} \in G$. Then there exists a unique Euler-Lagrange type additive mapping $E L: G \rightarrow X$ such that

$$
\|f(x)-E L(x)\| \leq \frac{\kappa}{|4|}\left\{\xi\left(\left|\frac{x}{r_{i}}\right|\right)+\xi\left(\left|\frac{x}{r_{j}}\right|\right)\right\} .
$$

Proof Defining $\zeta: G^{m} \rightarrow[0, \infty)$ by $\varphi\left(x_{1}, \ldots, x_{m}\right):=\kappa\left(\sum_{k=1}^{m} \xi\left(\left|x_{k}\right|\right)\right)$, then we have

$$
\lim _{n \rightarrow \infty}|2|^{n} \varphi\left(\frac{x_{1}}{2^{n}}, \ldots, \frac{x_{m}}{2^{n}}\right) \leq \lim _{n \rightarrow \infty}\left(|2| \xi\left(\frac{1}{|2|}\right)\right)^{n} \varphi\left(x_{1}, \ldots, x_{m}\right)=0
$$

for all $x_{1}, \ldots, x_{m} \in G$. On the other hand,

$$
\begin{aligned}
\Theta(x)= & \lim _{n \rightarrow \infty} \max \left\{| 2 | ^ { k } \operatorname { m a x } \left\{\operatorname { m a x } \left\{\varphi_{i, j}\left(\frac{x}{2^{k+2} r_{i}},-\frac{x}{2^{k+2} r_{j}}\right), \varphi_{i, j}\left(\frac{x}{2^{k+2} r_{i}}, 0\right),\right.\right.\right. \\
& \left.\varphi_{i, j}\left(0,-\frac{x}{2^{k+2} r_{j}}\right)\right\}, \frac{1}{|2|} \max \left\{\varphi_{i, j}\left(\frac{x}{2^{k+1} r_{i}}, \frac{x}{2^{n+1} r_{j}}\right), \varphi_{i, j}\left(\frac{x}{2^{k+1} r_{i}}, 0\right),\right. \\
& \left.\left.\left.\varphi_{i, j}\left(0, \frac{x}{2^{k+1} r_{j}}\right)\right\}\right\} \mid 0 \leq k<n\right\} \\
= & \max \left\{\max \left\{\varphi_{i, j}\left(\frac{x}{4 r_{i}},-\frac{x}{4 r_{j}}\right), \varphi_{i, j}\left(\frac{x}{4 r_{i}}, 0\right), \varphi_{i, j}\left(0,-\frac{x}{4 r_{j}}\right)\right\},\right. \\
& \left.\frac{1}{|2|} \max \left\{\varphi_{i, j}\left(\frac{x}{2 r_{i}}, \frac{x}{2 r_{j}}\right), \varphi_{i, j}\left(\frac{x}{2 r_{i}}, 0\right), \varphi_{i, j}\left(0, \frac{x}{2 r_{j}}\right)\right\}\right\} \\
= & \frac{\kappa}{|4|}\left\{\xi\left(\left|\frac{x}{r_{i}}\right|\right)+\xi\left(\left|\frac{x}{r_{j}}\right|\right)\right\}
\end{aligned}
$$

for all $x \in G$, exists. Also

$$
\begin{aligned}
\lim _{p \rightarrow \infty} \lim _{n \rightarrow \infty} \max \left\{| 2 | ^ { k } \operatorname { m a x } \left\{\operatorname { m a x } \left\{\varphi_{i, j}\left(\frac{x}{2^{k+2} r_{i}},-\frac{x}{2^{k+2} r_{j}}\right), \varphi_{i, j}\left(\frac{x}{2^{k+2} r_{i}}, 0\right),\right.\right.\right. \\
\left.\varphi_{i, j}\left(0,-\frac{x}{2^{k+2} r_{j}}\right)\right\}, \frac{1}{|2|} \max \left\{\varphi_{i, j}\left(\frac{x}{2^{k+1} r_{i}}, \frac{x}{2^{n+1} r_{j}}\right), \varphi_{i, j}\left(\frac{x}{2^{k+1} r_{i}}, 0\right),\right. \\
\left.\left.\left.\varphi_{i, j}\left(0, \frac{x}{2^{k+1} r_{j}}\right)\right\}\right\} \mid p \leq k<n+p\right\} \\
=\lim _{p \rightarrow \infty}|2|^{p} \max \left\{\max \left\{\varphi_{i, j}\left(\frac{x}{2^{p+2} r_{i}},-\frac{x}{2^{p+2} r_{j}}\right), \varphi_{i, j}\left(\frac{x}{2^{p+2} r_{i}}, 0\right), \varphi_{i, j}\left(0,-\frac{x}{2^{p+2} r_{j}}\right)\right\},\right. \\
\left.\frac{1}{|2|} \max \left\{\varphi_{i, j}\left(\frac{x}{2^{p+1} r_{i}}, \frac{x}{2^{p+1} r_{j}}\right), \varphi_{i, j}\left(\frac{x}{2^{p+1} r_{i}}, 0\right), \varphi_{i, j}\left(0, \frac{x}{2^{p+1} r_{j}}\right)\right\}\right\} \\
=0 .
\end{aligned}
$$

Applying Theorem 3.1, we get the desired result.

Theorem 3.2 Let $\varphi: G^{m} \rightarrow[0,+\infty)$ be a function such that

$$
\lim _{n \rightarrow \infty} \frac{\varphi\left(2^{n} x_{1}, \ldots, 2^{n} x_{m}\right)}{|2|^{n}}=0
$$


for all $x_{1}, \ldots, x_{m} \in G$ and let for each $x \in G$ the limit

$$
\begin{aligned}
\Theta(x)= & \lim _{n \rightarrow \infty} \max \left\{\frac { 1 } { | 2 | ^ { k } } \operatorname { m a x } \left\{\operatorname { m a x } \left\{\varphi_{i, j}\left(\frac{2^{k-1} x}{r_{i}},-\frac{2^{k-1} x}{r_{j}}\right),\right.\right.\right. \\
& \left.\varphi_{i, j}\left(\frac{2^{k-1} x}{r_{i}}, 0\right), \varphi_{i, j}\left(0,-\frac{2^{k-1} x}{r_{j}}\right)\right\}, \\
& \left.\left.\frac{1}{|2|} \max \left\{\varphi_{i, j}\left(\frac{2^{k} x}{r_{i}}, \frac{2^{k} x}{r_{j}}\right), \varphi_{i, j}\left(\frac{2^{k} x}{r_{i}}, 0\right), \varphi_{i, j}\left(0, \frac{2^{k} x}{r_{j}}\right)\right\}\right\} \mid 0 \leq k<n\right\}
\end{aligned}
$$

exist. Suppose that $f: G \rightarrow X$ is a mapping with $f(0)=0$ satisfying (3.3). Then, the limit $E L(x):=\lim _{n \rightarrow \infty} \frac{f\left(2^{n} x\right)}{2^{n}}$ exists for all $x \in G$ and defines an Euler-Lagrange type additive mapping $E L: G \rightarrow X$, such that

$$
\|f(x)-E L(x)\| \leq \frac{1}{|2|} \Theta(x)
$$

Moreover, if

$$
\begin{aligned}
& \lim _{p \rightarrow \infty} \lim _{n \rightarrow \infty} \max \left\{\frac { 1 } { | 2 | ^ { k } } \operatorname { m a x } \left\{\operatorname { m a x } \left\{\varphi_{i, j}\left(\frac{2^{k-1} x}{r_{i}},-\frac{2^{k-1} x}{r_{j}}\right),\right.\right.\right. \\
& \left.\varphi_{i, j}\left(\frac{2^{k-1} x}{r_{i}}, 0\right), \varphi_{i, j}\left(0,-\frac{2^{k-1} x}{r_{j}}\right)\right\}, \\
& \left.\left.\frac{1}{|2|} \max \left\{\varphi_{i, j}\left(\frac{2^{k} x}{r_{i}}, \frac{2^{k} x}{r_{j}}\right), \varphi_{i, j}\left(\frac{2^{k} x}{r_{i}}, 0\right), \varphi_{i, j}\left(0, \frac{2^{k} x}{r_{j}}\right)\right\}\right\} \mid p \leq k<n+p\right\}=0
\end{aligned}
$$

then EL is the unique Euler-Lagrange type additive mapping satisfying (3.13).

Proof It follows from (2.16) that

$$
\begin{aligned}
& \left\|\frac{f(2 x)}{2}-f(x)\right\| \\
& \leq \frac{1}{|2|} \max \left\{\max \left\{\varphi_{i, j}\left(\frac{x}{2 r_{i}},-\frac{x}{2 r_{j}}\right), \varphi_{i, j}\left(\frac{x}{2 r_{i}}, 0\right), \varphi_{i, j}\left(0,-\frac{x}{2 r_{j}}\right)\right\},\right. \\
& \left.\quad \frac{1}{|2|} \max \left\{\varphi_{i, j}\left(\frac{x}{r_{i}}, \frac{x}{r_{j}}\right), \varphi_{i, j}\left(\frac{x}{r_{i}}, 0\right), \varphi_{i, j}\left(0, \frac{x}{r_{j}}\right)\right\}\right\}
\end{aligned}
$$

for all $x \in G$. Replacing $x$ by $2^{n} x$ in (3.14), we obtain

$$
\begin{aligned}
\left\|\frac{f\left(2^{n+1} x\right)}{2^{n+1}}-\frac{f\left(2^{n} x\right)}{2^{n}}\right\| \\
\leq \frac{1}{|2|^{n+1}} \max \left\{\max \left\{\varphi_{i, j}\left(\frac{2^{n-1} x}{r_{i}},-\frac{2^{n-1} x}{r_{j}}\right), \varphi_{i, j}\left(\frac{2^{n-1} x}{r_{i}}, 0\right), \varphi_{i, j}\left(0,-\frac{2^{n-1} x}{r_{j}}\right)\right\},\right. \\
\left.\quad \frac{1}{|2|} \max \left\{\varphi_{i, j}\left(\frac{2^{n} x}{r_{i}}, \frac{2^{n} x}{r_{j}}\right), \varphi_{i, j}\left(\frac{2^{n} x}{r_{i}}, 0\right), \varphi_{i, j}\left(0, \frac{2^{n} x}{r_{j}}\right)\right\}\right\} .
\end{aligned}
$$


It follows from (3.11) and (3.15) that the sequence $\left\{\frac{f\left(2^{n} x\right)}{2^{n}}\right\}_{n \geq 1}$ is convergent. Set

$$
E L(x):=\lim _{n \rightarrow \infty} \frac{f\left(2^{n} x\right)}{2^{n}}
$$

On the other hand, it follows from (3.15) that

$$
\begin{aligned}
\| & \frac{f\left(2^{p} x\right)}{2^{p}}-\frac{f\left(2^{q} x\right)}{2^{q}} \| \\
= & \left\|\sum_{k=p}^{q-1} \frac{f\left(2^{k+1} x\right)}{2^{k+1}}-\frac{f\left(2^{k} x\right)}{2^{k}}\right\| \\
\leq & \max \left\{\left\|\frac{f\left(2^{k+1} x\right)}{2^{k+1}}-\frac{f\left(2^{k} x\right)}{2^{k}}\right\| ; p \leq k \leq q-1\right\} \\
\leq & \max \left\{\frac { 1 } { | 2 | ^ { k + 1 } } \operatorname { m a x } \left\{\max \left\{\varphi_{i, j}\left(\frac{2^{k-1} x}{r_{i}},-\frac{2^{k-1} x}{r_{j}}\right), \varphi_{i, j}\left(\frac{2^{k-1} x}{r_{i}}, 0\right), \varphi_{i, j}\left(0,-\frac{2^{k-1} x}{r_{j}}\right)\right\},\right.\right. \\
& \left.\left.\frac{1}{|2|} \max \left\{\varphi_{i, j}\left(\frac{2^{k} x}{r_{i}}, \frac{2^{k} x}{r_{j}}\right), \varphi_{i, j}\left(\frac{2^{k} x}{r_{i}}, 0\right), \varphi_{i, j}\left(0, \frac{2^{k} x}{r_{j}}\right)\right\}\right\} \mid p \leq k<q\right\}
\end{aligned}
$$

for all $x \in G$ and all nonnegative integers $p, q$ with $q>p \geq 0$. Letting $p=0$ and passing the limit $q \rightarrow \infty$ in the last inequality and using (3.12), we obtain (3.13). The rest of the proof is similar to the proof of Theorem 3.1.

Corollary 3.2 Let $\xi:[0, \infty) \rightarrow[0, \infty)$ be a function satisfying

$$
\xi(|2 t|) \leq \xi(|2|) \xi(t) \quad(t \geq 0) \quad \xi(|2|)<|2| .
$$

Let $\kappa>0$ and $f: G \rightarrow X$ be a mapping with $f(0)=0$ satisfying the following inequality (3.9). Then there exists a unique Euler-Lagrange type additive mapping $E L: G \rightarrow X$ such that

$$
\begin{aligned}
\|f(x)-E L(x)\| & \leq \frac{\kappa}{|2|} \max \left\{\xi\left(\left|\frac{x}{2 r_{i}}\right|\right)+\xi\left(\left|\frac{x}{2 r_{j}}\right|\right), \frac{1}{|2|} \xi\left(\left|\frac{x}{r_{i}}\right|\right)+\xi\left(\left|\frac{x}{r_{j}}\right|\right)\right\} \\
& =\frac{\kappa}{|2|}\left[\xi\left(\left|\frac{x}{2 r_{i}}\right|\right)+\xi\left(\left|\frac{x}{2 r_{j}}\right|\right)\right] .
\end{aligned}
$$

Proof Defining $\zeta: G^{m} \rightarrow[0, \infty)$ by $\varphi\left(x_{1}, \ldots, x_{m}\right):=\kappa\left(\sum_{k=1}^{m} \xi\left(\left|x_{k}\right|\right)\right)$, then, we have

$$
\lim _{n \rightarrow \infty} \frac{\varphi\left(2^{n} x_{1}, \ldots, 2^{n} x_{m}\right)}{|2|^{n}} \leq \lim _{n \rightarrow \infty}\left(\frac{\xi(|2|)}{|2|}\right)^{n} \varphi\left(x_{1}, \ldots, x_{m}\right)=0
$$

for all $x_{1}, \ldots, x_{m} \in G$. On the other hand,

$$
\begin{aligned}
\Theta(x)= & \lim _{n \rightarrow \infty} \max \left\{\frac { 1 } { | 2 | ^ { k } } \operatorname { m a x } \left\{\operatorname { m a x } \left\{\varphi_{i, j}\left(\frac{2^{k-1} x}{r_{i}},-\frac{2^{k-1} x}{r_{j}}\right),\right.\right.\right. \\
& \left.\varphi_{i, j}\left(\frac{2^{k-1} x}{r_{i}}, 0\right), \varphi_{i, j}\left(0,-\frac{2^{k-1} x}{r_{j}}\right)\right\}, \\
& \left.\left.\frac{1}{|2|} \max \left\{\varphi_{i, j}\left(\frac{2^{k} x}{r_{i}}, \frac{2^{k} x}{r_{j}}\right), \varphi_{i, j}\left(\frac{2^{k} x}{r_{i}}, 0\right), \varphi_{i, j}\left(0, \frac{2^{k} x}{r_{j}}\right)\right\}\right\} \mid 0 \leq k<n\right\}
\end{aligned}
$$




$$
\begin{aligned}
= & \max \left\{\max \left\{\varphi_{i, j}\left(\frac{x}{2 r_{i}},-\frac{x}{2 r_{j}}\right), \varphi_{i, j}\left(\frac{x}{2 r_{i}}, 0\right), \varphi_{i, j}\left(0,-\frac{x}{2 r_{j}}\right)\right\},\right. \\
& \left.\frac{1}{|2|} \max \left\{\varphi_{i, j}\left(\frac{x}{r_{i}}, \frac{x}{r_{j}}\right), \varphi_{i, j}\left(\frac{x}{r_{i}}, 0\right), \varphi_{i, j}\left(0, \frac{x}{r_{j}}\right)\right\}\right\}
\end{aligned}
$$

for all $x \in G$, exists. Also

$$
\begin{aligned}
& \lim _{p \rightarrow \infty} \lim _{n \rightarrow \infty} \max \left\{\frac { 1 } { | 2 | ^ { k } } \operatorname { m a x } \left\{\operatorname { m a x } \left\{\varphi_{i, j}\left(\frac{2^{k-1} x}{r_{i}},-\frac{2^{k-1} x}{r_{j}}\right),\right.\right.\right. \\
& \left.\varphi_{i, j}\left(\frac{2^{k-1} x}{r_{i}}, 0\right), \varphi_{i, j}\left(0,-\frac{2^{k-1} x}{r_{j}}\right)\right\}, \\
& \left.\left.\frac{1}{|2|} \max \left\{\varphi_{i, j}\left(\frac{2^{k} x}{r_{i}}, \frac{2^{k} x}{r_{j}}\right), \varphi_{i, j}\left(\frac{2^{k} x}{r_{i}}, 0\right), \varphi_{i, j}\left(0, \frac{2^{k} x}{r_{j}}\right)\right\}\right\} \mid p \leq k<n+p\right\}=0 .
\end{aligned}
$$

Applying Theorem 3.2, we get the desired result.

Remark 3.1 We remark that if $\xi(|2|)=0$, then $\xi=0$ identically, and so $f$ is itself additive. Thus, for the nontrivial $\xi$, we observe that $\xi(|2|) \neq 0$ and

$$
1 \leq \xi(|1|) \leq \xi(|2|) \xi\left(\frac{1}{|2|}\right) \leq|2| \xi\left(\frac{1}{|2|}\right)
$$

implies that $\frac{1}{|2|} \leq \xi\left(\frac{1}{|2|}\right)$.

\section{Competing interests}

The authors declare that they have no competing interests.

\section{Authors' contributions}

All authors conceived of the study participated in its design and coordination, drafted the manuscript, participated in the sequence alignment, and read and approved the final manuscript.

\section{Author details}

'Department of Mathematics, College of Sciences, Yasouj University, 75914-353 Yasouj, Iran. ${ }^{2}$ Department of Mathematics, College of Natural Science, University of Seoul, Seoul, Korea. ${ }^{3}$ Department of Mathematics, Daejin University, Pocheon, Kyeonggi 487-711, Korea.

\section{Acknowledgement}

Dong Yun Shin was supported by the 2011 sabbatical year research grant of the University of Seoul.

\section{Received: 3 February 2012 Accepted: 2 July 2012 Published: 19 July 2012}

\section{References}

1. Arriola, LM, Beyer, WA: Stability of the Cauchy functional equation over p-adic fields. Real Anal. Exch. 31, 125-132 (2005/06)

2. Balcerowski, M: On the functional equation $f(x+g(y))-f(y+g(y))=f(x)-f(y)$ on groups. Aequ. Math. 78, 247-255 (2009)

3. Cho, YJ, Park, C, Saadati, R: Functional inequalities in non-Archimedean in Banach spaces. Appl. Math. Lett. 60 1994-2002 (2010)

4. Cho, YJ, Saadati, R: Lattice non-Archimedean random stability of ACQ functional equation. Adv. Differ. Equ. 2011, 31 (2011)

5. Cholewa, PW: Remarks on the stability of functional equations. Aequ. Math. 27, 76-86 (1984)

6. Czerwik, S: Functional Equations and Inequalities in Several Variables. World Scientific, River Edge (2002)

7. Ebadian, A, Ghobadipour, N, Gordji, ME: A fixed point method for perturbation of bimultipliers and Jordan bimultipliers in $C^{*}$-ternary algebras. J. Math. Phys. 51(10), 103508 (2010). doi:10.1063/1.3496391

8. Ebadian, A, Kaboli Gharetapeh, S, Eshaghi Gordji, M: Nearly Jordan *-homomorphisms between unital $C^{*}$-algebras. Abstr. Appl. Anal. 2011, Article ID 513128 (2011). doi:10.1155/2011/513128

9. Ebadian, A, Najati, A, Eshaghi Gordji, M: On approximate additive-quartic and quadratic-cubic functional equations in two variables on abelian groups. Results Math. 58(1-2), 39-53 (2010) 
10. Eshaghi Gordji, M: Nearly ring homomorphisms and nearly ring derivations on non-Archimedean Banach algebras. Abstr. Appl. Anal. 2010, Article ID 393247 (2010). doi:10.1155/2010/393247

11. Eshaghi Gordji, M: Stability of a functional equation deriving from quartic and additive functions. Bull. Korean Math. Soc. 47(3), 491-502 (2010)

12. Eshaghi Gordji, M: Stability of an additive-quadratic functional equation of two variables in F-spaces. J. Nonlinear Sci. Appl. 2(4), 251-259 (2009)

13. Eshaghi Gordji, M, Abbaszadeh, S, Park, C: On the stability of generalized mixed type quadratic and quartic functional equation in quasi-Banach spaces. J. Inequal. Appl. 2009, Article ID 153084 (2009)

14. Eshaghi Gordji, M, Alizadeh, Z: Stability and superstability of ring homomorphisms on non-Archimedean Banach algebras. Abstr. Appl. Anal. 2011, Article ID 123656 (2011)

15. Găvruta, P: A generalization of the Hyers-Ulam-Rassias stability of approximately additive mappings. J. Math. Anal. Appl. 184, 431-436 (1994)

16. Gselmann, E, Maksa, G: Stability of the parametric fundamental equation of information for nonpositive parameters. Aequ. Math. 78, 271-282 (2009)

17. Hyers, DH: On the stability of the linear functional equation. Proc. Natl. Acad. Sci. USA 27, 222-224 (1941)

18. Mihet, D, Radu, V: On the stability of the additive Cauchy functional equation in random normed spaces. J. Math. Anal. Appl. 343, 567-572 (2008)

19. Najati, A, Kang, Jl, Cho, YJ: Local stability of the pexiderized Cauchy and Jensen's equations in fuzzy spaces. J. Inequal. Appl. 2011, 78 (2011)

20. Najati, A, Cho, YJ: Generalized Hyers-Ulam stability of the pexiderized Cauchy functional equation in non-Archimedean spaces. Fixed Point Theory Appl. 2011, Article ID 309026 (2011)

21. Najati, A, Park, C: Stability of a generalized Euler-Lagrange type additive mapping and homomorphisms in $C^{*}$-algebras. J. Nonlinear Sci. Appl. 3(2), 134-154 (2010)

22. Park, C: Fuzzy stability of a functional equation associated with inner product spaces. Fuzzy Sets Syst. 160, 1632-1642 (2009)

23. Park, C: Generalized Hyers-Ulam-Rassias stability of $n$-sesquilinear-quadratic mappings on Banach modules over $C^{*}$-algebras. J. Comput. Appl. Math. 180, 279-291 (2005)

24. Rassias, TM: On the stability of the linear mapping in Banach spaces. Proc. Am. Math. Soc. 72, 297-300 (1978)

25. Rassias, TM: On the stability of the quadratic functional equation and its applications. Stud. Univ. Babeş-Bolyai, Math. XLIII, 89-124 (1998)

26. Rassias, TM: The problem of S. M. Ulam for approximately multiplicative mappings. J. Math. Anal. Appl. 246, 352-378 (2000)

27. Rassias, TM: On the stability of functional equations in Banach spaces. J. Math. Anal. Appl. 251, 264-284 (2000)

28. Rassias, TM: On the stability of functional equations and a problem of Ulam. Acta Appl. Math. 62, 23-130 (2000)

29. Rassias, TM, Šemrl, P: On the Hyers-Ulam stability of linear mappings. J. Math. Anal. Appl. 173, 325-338 (1993)

30. Rassias, TM, Shibata, K: Variational problem of some quadratic functionals in complex analysis. J. Math. Anal. Appl. 228, 234-253 (1998)

31. Saadati, R, Cho, YJ, Vahidi, J: The stability of the quartic functional equation in various spaces. Comput. Math. Appl. 60 1994-2002 (2010)

32. Saadati, R, Vaezpour, M, Cho, YJ: A note to paper 'On the stability of cubic mappings and quartic mappings in random normed spaces'. J. Inequal. Appl. 2009, Article ID 214530 (2009). doi:10.1155/2009/214530

33. Saadati, R, Zohdi, MM, Vaezpour, SM: Nonlinear L-random stability of an ACQ functional equation. J. Inequal. Appl. 2011, Article ID 194394 (2011). doi:10.1155/2011/194394

34. Ulam, SM: Problems in Modern Mathematics. Science ed., Chapter VI. Wiley, New York (1940)

doi:10.1186/1687-1847-2012-111

Cite this article as: Azadi Kenary et al.: Non-Archimedean Hyers-Ulam-Rassias stability of $m$-variable functional equation. Advances in Difference Equations 2012 2012:111.

\section{Submit your manuscript to a SpringerOpen ${ }^{\ominus}$ journal and benefit from:}

- Convenient online submission

Rigorous peer review

- Immediate publication on acceptance

- Open access: articles freely available online

- High visibility within the field

- Retaining the copyright to your article 